\title{
Interstellar Overdrive
}

\author{
Paul Sutter
}

\section{Extraterrestrial: The First Sign of Intelligent Life Beyond Earth}

by Avi Loeb

Houghton Mifflin Harcourt, 240 pp., \$14.99.

$\mathrm{T}$ HE ORIGINS OF 'Oumuamua, the first known interstellar visitor to the solar system, are a mystery. Among the explanations for the object's appearance that have been advanced within the scientific community, the hypothesis proposed by the astrophysicist Avi Loeb is perhaps the most striking and unusual. Loeb's claims are striking not only for the ideas they embody-in particular, that 'Oumuamua is an artifact of alien technology-but also for his steadfast insistence on their validity.

Despite the passage of several years since 'Oumuamua's fleeting visit to the solar system, Loeb's views have not wavered. In Extraterrestrial: The First Sign of Intelligent Life Beyond Earth, he attempts to make the case not only for 'Oumuamua as a lightsail spacecraft created by extraterrestrial intelligence, but also for the notion of extraterrestrial intelligence itself. Indeed, the book ventures far beyond 'Oumuamua, examining the history of the search for extraterrestrial intelligence, the present-day hunt for biosignatures on other worlds, and the implications for humanity of discovering life elsewhere in the universe.

At the center of everything is Loeb himself. And this is where the problems begin.

$\mathrm{T}$ HE OBJECT KNOWN as 'Oumuamua was first spotted on October 19, 2017, by astronomer Robert Weryk using the Pan-STARRS telescope in Hawaii. ${ }^{1}$ At the time of its discovery, 'Oumuamua was 33 million kilometers, or 0.22 astronomical units (AU), away from the earth and already traveling along a solar-escape trajectory. It was recognized by astronomers as an extrasolar body due to the extreme eccentricity of its orbit and velocity of roughly 26 kilometers per second relative to the sun.

It soon became apparent to observers that 'Oumuamua was quite unlike any object ever seen in the solar system. At between 100 and 1,000 meters in length, and with an average diameter of around 110 meters, 'Oumuamua is more elongated in comparison to other known similarly sized objects. It is either cigar shaped or a flattened oval, with a long-to-short axis ratio of at least 6 to 1. Based on variations in its light curve, 'Oumuamua appears to tumble around its shortest axis every eight hours, a variation seen in only a handful of objects. Although 'Oumuamua passed within $0.25 \mathrm{AU}$ of the sun, its infrared signature was too faint to be detected by the Spitzer Space Telescope, indicating that it must be far more shiny and reflective than the comets of the solar system. When it first appeared in the solar system, 'Oumuamua was relatively stationary with respect to the local standard of rest, the mean motion of stars within 100 parsecs of the sun. By contrast, the velocities of almost all nearby stars are roughly five times greater. The fact that 'Oumuamua was even observable at all using current technology suggests it may be just one member of a vast population of interstellar nomads-a population several orders of magnitude larger than previously estimated.

'Oumuamua's most important characteristic for the purposes of Loeb's hypothesis is its nongravitational acceleration. As 'Oumuamua raced away from Earth, it deviated from a Newtonian trajectory, with the magnitude of the acceleration dropping off in relation to the square of its distance from the sun. A deviation like this has been previously observed for comets and is thought to be the result of outgassing caused by solar heating. In the case of comets, this process releases a cloud of gas and dust that surrounds the object. No such cloud was observed around 'Oumuamua, suggesting it may contain little, if any, dust or fine-grained material.

For Loeb, the strangeness of 'Oumuamua is highly suggestive. In Extraterrestrial, he refers to 'Oumuamua several times as a "one-in-a-million" object. ${ }^{2}$ Near the end of the book he quotes odds of "roughly one in one trillion, give or take, that "Oumuamua was a unique rock." ${ }^{3}$ Although Loeb makes no mention of how he arrived at these estimates, he concludes that 'Oumuamua is not a natural object, but rather a lightsail: a small spacecraft propelled by reflected starlight or laser light.

Loeb's lightsail hypothesis is a neat explanation for four reasons: first, the object's non-Newtonian acceleration 
is accounted for by lightsail propulsion; second, its shininess can be attributed to the lightsail reflecting rather than absorbing radiation; third, its shape reflects the fact that the best lightsails are broad and thin; and, finally, its existence and position prior to entering the solar system becomes purposeful and explainable.

Despite being presented as the main subject of Extraterrestrial, less than ten percent of the book is devoted to Loeb's hypothesis and technical analysis. One possible explanation for the small portion of the text spent discussing 'Oumuamua is that any further analysis might reveal the numerous inconsistencies in Loeb's arguments and undermine the remainder of the book.

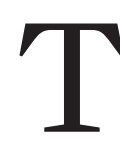

He velocity of 'Oumuamua and its comparatively small size meant that it was only observable by astronomers for eleven days. All that we knowand all that we will ever know-about 'Oumuamua was gathered during that brief period. Despite the limited dataset, researchers have still managed to learn much about the object. These findings do little to bolster the hypothesis described in Extraterrestrial.

Contrary to Loeb's claims, 'Oumuamua is not, in fact, unusually shiny. Instead it has an albedo of around 0.1, where 0 corresponds to an object that absorbs all solar radiation. This is a similar result to those of other objects in the solar system and is not nearly reflective enough for an effective lightsail. This point alone undermines all the calculations on which Loeb bases his hypothesis.

'Oumuamua approached the solar system at close to the local standard of rest, but, despite what Loeb suggests in Extraterrestrial, there was nothing unusual about its trajectory. During those eleven days of observations, 'Oumuamua behaved exactly as astronomers predicted that detectable interstellar objects would behave. ${ }^{4}$

Elsewhere in the book, Loeb argues that no model of planetary system mass loss could yield sufficiently many and varied interstellar objects such that a detectable example might one day appear in the solar system. ${ }^{5}$ The underlying size distribution of interstellar objects is unknown. Astronomers simply have no idea. As a result, any claims about the rarity of 'Oumuamua must be treated as speculation.

On the topic of 'Oumuamua's nongravitational acceleration and apparent lack of outgassing, Loeb fails to include an important caveat: 'Oumuamua was extremely difficult to observe and the surveys undertaken were far from comprehensive. It is entirely possible that any outgassing was missed by astronomers, or that outgassing proceeded through an unobservable channel, or even that solar heating played a role. There are, in fact, any number of plausible explanations.

'Oumuamua's tumbling motion also poses a problem for Loeb's hypothesis. His lightsail calculations cannot account for this motion and, to arrive at the right size and density for the object, he must assume that the sail always remained perpendicular to the sun. A tumbling lightsail would periodically vanish-not just dim-relative to the vantage point of astronomers on Earth. Over the course of eleven days of observations, these variations would also change due to the earth's orbit. There is no known lightsail configuration that can satisfy all these requirements.

In weighing up different explanations for 'Oumuamua's low velocity relative to the local standard of rest, Loeb speculates that intelligent extraterrestrials might deploy a vast array of such objects as waypoints or buoys.

[M]aybe it was more like a lighthouse-or, more simply, a signpost or navigation marker. A vast network of such buoys could act as a communication grid. Or it could be used as a trip wire, an alert system triggered when one of them was knocked out of [local standard of rest]. ${ }^{6}$

These suggestions illustrate the main weakness of arguments based on alien technology: all manner of intentions and capabilities can be attributed to an intelligent extraterrestrial actor in order to plug a hole in a theory or explain away mysteries, no matter how outlandish, convoluted, or contradictory they may seem.

One [possibility] is that 'Oumuamua's makers intentionally targeted our inner solar system; the other is that 'Oumuamua is a piece of space junk that happened upon us (or we upon it). Either of these interpretations could be accurate regardless of whether the civilization that created 'Oumuamua still exists today. ${ }^{7}$

$\mathrm{L}$ OEB IS THE chairman of the advisory committee for Breakthrough Starshot, a project initiated in 2016 by the entrepreneur Yuri Milner that aims to "demonstrate proof of concept for ultra-fast light-driven nanocrafts, and lay the foundations for a first launch to Alpha Centauri within the next generation." ${ }^{8}$ As Loeb readily admits in Extraterrestrial, aliens, lightsails, and interstellar travel were already on his mind when 'Oumuamua first appeared. The realization that the object was the product of alien technology followed naturally.

There is little, if any, support among the astronomical community for Loeb's claims concerning 'Oumuamua. Loeb, it should be noted, has comparatively little experience studying similarly sized objects in the solar system. Since he first began publicizing these claims in 2018, ${ }^{9}$ they have been refuted on numerous occasions. An international team of experts was unequivocal, maintaining that their observations are "consistent with a purely natural origin for "Oumuamua." 10

In Extraterrestrial, Loeb frequently expresses bewilderment that colleagues fail to recognize the validity of his arguments. In doing so, he often conflates disagreement with dismissal. The arguments raised against his hypothe- 
sis for 'Oumuamua are exaggerated unfairly and presented as ruling out the existence of aliens. "Given the ubiquity of habitable planets," he remarks, "it is the height of arrogance to conclude that we are unique."11 But this is putting words in the mouths of his critics. Reasonable criticisms of his hypothesis are brushed aside.

Against this backdrop, Loeb proposes a bet along similar lines to Blaise Pascal's famous wager about the existence of God: "In much the same way, I would argue, humanity bets its future on whether 'Oumuamua is extraterrestrial technology or not."12 Loeb views his wager as far less demanding than that of Pascal, requiring "only a modest leap of hope-specifically, hope for more scientific evidence," and that to posit "Oumuamua is extraterrestrial technology requires only the belief in an intelligence other than our own."13 Belief in the existence of aliens, according to Loeb, is essential to motivate the search for intelligent life elsewhere in the universe. Such a discovery, he believes, has the potential to transform humanity.

It is not too much to presume that such a discovery would also affect the way we behave and how we interact with each other, because we might come to feel as though we are a part of a single, unified team, humanity, and stop worrying and warring over mundane issues like geographical borders and separate economies. ${ }^{14}$

In attempting to replicate Pascal's wager, Loeb seems unaware that his version is vulnerable to the same criticisms leveled at its predecessor. 'Oumuamua's wager offers no proof of the existence of aliens, admits no other possibilities outside this false dichotomy, cannot guarantee the positive outcomes that it promises, and ignores the costs associated with these beliefs.

\section{$\mathrm{L}$}

OEB IS A FINE writer with a gift for explaining complex scientific concepts in clear and straightforward terms. But beyond the granular level, Extraterrestrial is a mess. After the discussion concerning 'Oumuamua is dealt with in short order, the book meanders from one topic to another with little apparent concern for cohesion or momentum. Lengthy divagations on black holes and the first stars, to cite just two examples, appear to be included for no reason other than that Loeb has published papers on those topics.

The majority of Extraterrestrial is devoted to Loeb's musings on a wide range of subjects, including but not limited to the future of humanity, the nature of aliens, Fermi's paradox, Gutenberg DNA printers, and panspermia. These discussions have little scholarly content and lead nowhere. "Even though life as we know it and life as we do not know it may exist on numerous other planets," Loeb remarks at one point, "it is most likely that we will encounter relics of extraterrestrial technologies before establishing contact with any living civilization."15 Rather than offer any further reasoning or evidence to support this assertion, Loeb simply moves on to another topic. This approach is by no means uncommon.

Although Extraterrestrial is poorly organized and frustrating to navigate, Loeb still raises some important points. His claims that students are incentivized to follow mainstream research or risk damaging their careers merit further consideration. He is also on solid ground when he suggests that scientists should appear more often in the media and that the public should be made more aware of what he describes as, "the messy reality of science-that it's full of starts and stops and dead ends."16 Loeb is far from alone when he criticizes the emphasis on unproven string and supersymmetry theories in particle physics, but his points are still well made. Even when he disparages his fellow astronomers-at one point labeling any survey not dedicated to the search for extraterrestrial life "low-key" and brushing off many areas of astrophysics as "fashionable thought bubbles"-his tone and vision for the future is relentlessly optimistic and hopeful. ${ }^{17}$

Although these points are mentioned prominently, there is little, if any, deeper discussion. This is a disappointing outcome for many reasons. Chief among them is, in fact, the reason why Loeb squanders these opportunities. Each time Extraterrestrial approaches a new topic, it is not long before the spotlight returns to the book's main character: Avi Loeb. Despite the nominal subject matter of the book, Loeb shoehorns in passages detailing the industriousness of his father and the unerring wisdom of his mother, the advice he offers his precocious daughters, the vacation spots that his family prefers, and the age of his house. Descriptions of parties Loeb attended with Stephen Hawking are included, along with accounts of his meetings with leading political and industrial figures. A weekend getaway to a secluded goat farm in the Negev is described in vivid detail.

The weather was cool and dry. I looked out on the goats that had been born the previous day. It was all very familiar, reminding me of the farm I had grown up on with my two sisters, Reli and Shoshi. Among my tasks had been collecting eggs and helping to corral the farm's just-born chicks when they escaped their cages. It was in this familiar setting that I typed out my plans for humanity's first interstellar probe using lightsail technology. ${ }^{18}$

Alongside the frequent diversions into Loeb's personal life, the book catalogues his many insights and notable accomplishments.

Well before I had a drawer for collecting ideas, let alone undergraduates, graduate students, and postdocs to share these ideas with, I was gathering them. They have served as seeds from which my own research has grown. To date, 
those seeds have yielded more than seven hundred published papers, six books (including the one in your hand), and a growing number of now-confirmed predictions touching on the birth of stars, the detection of planets beyond our solar system, and the properties of black holes. ${ }^{19}$

In Loeb's account, it is always his work that takes center stage. "My research on the cosmic dawn," he notes at one point, "contributed to the creation of a new field of study." Reading the book gives the impression that Loeb works in a vacuum. Any collaborators are relegated to the supporting cast, while his postdocs and graduate students merely help flesh out his ideas. Loeb's colleagues and fellow scientists are mentioned only for their reactions to his musings. "Freeman Dyson corresponded with me routinely about my research," Loeb recalls, adding that, "he had started showing interest in Starshot as well." "21 The hundreds of researchers who studied 'Oumuamua are subsumed into a collective "we," even though Loeb did not directly participate in any of the observations. At the end of the book, Loeb provides an "Additional Reading" section that contains a list of 206 papers, organized by chapter. Of these papers, all 206 list Loeb either as the sole author or as a contributor.

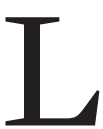

OEB MAY WELL be correct in claiming that 'Oumuamua is an artifact from a technologically advanced alien civilization. We will never know. But he does not make a convincing case in Extraterrestrial and the remainder of the book does not advance any useful scientific discussion.

Paul Sutter is a research professor in astrophysics at the Institute for Advanced Computational Science at Stony Brook University.

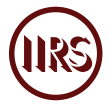

1. MPEC 2017-U181: COMET C/2017 U1 (PANSTARRS), International Astronomical Union Minor Planet Center.

2. Avi Loeb, Extraterrestrial: The First Sign of Intelligent Life Beyond Earth (New York: Houghton Mifflin Harcourt, 2021), 61,86 .

3. Loeb, Extraterrestrial, 176.

4. Michele Bannister et al., "The Natural History of 'Oumuamua," Nature Astronomy 3, no. 7 (2019): 594-602, doi:10.1038/s41550-019-0816-x.

5. Loeb, Extraterrestrial, 77.

6. Loeb, Extraterrestrial, 84.

7. Loeb, Extraterrestrial, 119.

8. "Starshot," Breakthrough Initiatives.

9. Shmuel Bialy and Abraham Loeb, "Could Solar Radiation Pressure Explain 'Oumuamua's Peculiar Acceleration?" The Astrophysical Journal Letters 868 (2018): L1, doi:10.3847/2041-8213/aaeda8.

10. Bannister et al., "Natural History."

11. Loeb, Extraterrestrial, 152.

12. Loeb, Extraterrestrial, 153.

13. Loeb, Extraterrestrial, 153.

14. Loeb, Extraterrestrial, 151-52.

15. Loeb, Extraterrestrial, 114.

16. Loeb, Extraterrestrial, 106.

17. Loeb, Extraterrestrial, 141, 99.

18. Loeb, Extraterrestrial, 60.

19. Loeb, Extraterrestrial, 94.

20. Loeb, Extraterrestrial, 114.

21. Loeb, Extraterrestrial, 60.

DOI: $10.37282 / 991819.21 .68$ 